\title{
Modulation of La Crosse virus infection in Aedes albopictus mosquitoes following larval exposure to coffee extracts
}

\author{
Nicole E. Eastep, Rachel E. Albert and Justin R. Anderson*
}

Radford Arbovirus and Medical Entomology Laboratory, Department of Biology, Radford University, Radford, VA, USA

Edited by:

Rubén Bueno-Marí, University of

Valencia, Spain

\section{Reviewed by:}

Ying Xu, West Virginia University, USA Harish Padmanabha, Yale University, USA

Roger Eritja, Consell Comarcal Del Baix Llobregat, Spain

\section{${ }^{*}$ Correspondence:}

Justin R. Anderson, Department of Biology, Radford University, Box 6931, Radford, VA 24142, USA.

e-mail: janderson152@radford.edu
The mosquito-borne La Crosse virus (LACV; Family Bunyaviridae) may cause encephalitis, primarily in children, and is distributed throughout much of the eastern United States. No antivirals or vaccines are available for LACV, or most other mosquito-borne viruses, and prevention generally relies on mosquito control. We sought to determine whether coffee extracts could interfere with LACV replication and vector mosquito development. Both regular and decaffeinated coffee demonstrated significant reductions in LACV replication in direct antiviral assays. This activity was not due to the presence of caffeine, which did not inhibit the virus life cycle. Aedes albopictus (Skuse; Diptera: Culicidae) mosquito larvae suffered near total mortality when reared in high concentrations of regular and decaffeinated coffee and in caffeine. Following larval exposure to sublethal coffee concentrations, adult $A$. albopictus mosquitoes had significantly reduced whole-body LACV titers 5 days post-infection, compared to larvae reared in distilled water. These results suggest that it may be possible to both control mosquito populations and alter the vector competence of mosquitoes for arthropod-borne viruses by introducing antiviral compounds into the larval habitat.

Keywords: arbovirus, mosquito larvicide, antiviral, La Crosse virus, Bunyaviridae

\section{INTRODUCTION}

La Crosse virus (LACV) is an arthropod-borne virus (arbovirus) in the California serogroup of the genus Orthobunyavirus, of the family Bunyaviridae and is distributed throughout most of the eastern United States. The virus is maintained in nature by transovarial passage from an infected female mosquito to her progeny via the egg and through an amplification cycle involving chipmunks and squirrels, which produce a high viremia capable of infecting other mosquitoes (Borucki et al., 2002). The natural vector is the eastern tree hole mosquito, Ochlerotatus triseriatus (Say), but other mosquitoes are also competent, including the following two introduced species: Aedes albopictus (Skuse; Grimstad et al., 1989) and Ochlerotatus japonicus (Theobald; Sardelis et al., 2002).

Human infections with LACV are common, though disease is rare, with an average of $\sim 70$ cases reported annually. Infection typically presents with a generic febrile illness that may progress to severe central nervous system involvement, including seizures, mental impairment, coma, and death. Sequelae may consist of persistent seizures for over 10 years and learning disabilities. Death is rare, occurring in $<2 \%$ of those displaying severe symptoms (Haddow and Odoi, 2009). There are no vaccines or antivirals used to treat infection with LACV and most other arboviruses. Prevention generally relies on avoidance of mosquito bites, either through personal protective measures or anti-mosquito insecticides.

Mosquito control generally uses either chemical insecticides or toxins derived from Bacillus species bacteria, although resistance to most control agents develops rather quickly (e.g., Cui et al., 2006; Paris et al., 2011). Novel mechanisms to prevent transmission of LACV and other mosquito-borne pathogens are therefore needed (Lambrechts et al., 2009; Luckhart et al., 2010). Environmentally friendly products derived from plants have been proffered as one source of mosquitocidal compounds, and many different extracts of plants have demonstrated mosquito larvicidal activity, though the effective concentrations vary widely among plant species and among extraction methods (Shaalan et al., 2005; Fallatah and Khater, 2010). Both caffeine and coffee extracts have been shown to inhibit the development of A. aegypti (L.) larvae (Laranja et al., 2003, 2006).

Further, plant extracts offer a potential source of antiviral compounds, and many have shown such activity against a broad spectrum of viruses (Jassim and Naji, 2003; Mukhtar et al., 2008). For example, coffee extracts from various sources have been shown to inhibit the replication of herpes simplex virus type 1 and poliovirus, and this antiviral activity did not require the presence of caffeine (Utsunomiya et al., 2008). In subsequent experiments, caffeine alone was shown to possess antiviral activity (Murayama et al., 2008), as did a newly identified compound in coffee, $N$-methyl-pyridinium formate (Tsujimoto et al., 2010).

In the present study, we investigated whether coffee extracts can interfere with mosquito development and LACV replication.

\section{MATERIALS AND METHODS ANTIVIRAL ASSAYS}

La Crosse virus was kindly provided by Sally Paulson (Virginia Polytechnic Institute and State University) and was isolated from O. triseriatus mosquitoes in southwest Virginia. Virus stocks were generated by inoculation of confluent monolayers of African green monkey kidney (Vero) cells, harvested from supernatants when $\sim 90 \%$ of the monolayer exhibited cytopathic effect, and titrated by plaque assay in Vero cells. 
Regular (Maxwell House Dark Roast) or decaffeinated (Great Value Classic Decaf Medium Roast) ground coffee beans were prepared according to the package directions by extracting one teaspoon coffee grounds with six fluid ounces deionized water ( $\sim 26.7 \mathrm{~g}$ coffee/L water) using a Mr. Coffee drip coffee maker. Whole coffee extracts were then sterilized by passage through a $0.22-\mu \mathrm{m}$ membrane filter and serially twofold diluted in Medium 199 (M199; Cellgro) supplemented with 5\% fetal bovine serum (FBS), amphotericin B $(25 \mu \mathrm{g} / 100 \mathrm{~mL})$, and gentamycin $(15 \mu \mathrm{g} / 100 \mathrm{~mL})$. Direct virucidal assays were performed by incubating $\sim 10^{5}$ plaque-forming units (PFUs) LACV in $800 \mu \mathrm{L}$ M199 with $200 \mu \mathrm{L}$ of each solution for $30 \mathrm{~min}$ at room temperature. Each dilution was then serially 10 -fold diluted in M199, and $400 \mu \mathrm{L}$ of each dilution was plated onto confluent Vero monolayers in sixwell plates (BD) for $1 \mathrm{~h}$ at $37^{\circ} \mathrm{C}$ and $5 \% \mathrm{CO}_{2}$. After incubating, the supernatant was removed by aspiration and replaced with M199 containing $0.8 \%$ gum tragacanth (MP Biomedicals) and FBS and antibiotics as above. Following a 3 -day incubation at $37^{\circ} \mathrm{C}$ and $5 \%$ $\mathrm{CO}_{2}$, the medium was poured off and the plates were stained in $1 \mathrm{mg} / \mathrm{mL}$ crystal violet in $10 \%$ formalin. Virus mortality was determined by comparing plaque numbers in coffee compound treated virus dilutions to virus incubated only in M199, which was set to $100 \%$ viability. Three independent replicates of each experiment were performed.

We also tested whether regular coffee could interfere with virus replication by adding undiluted coffee to Vero cells 1 or $24 \mathrm{~h}$ before or after infections with LACV as described above. Coffee was allowed to incubate on the Vero cells for $1 \mathrm{~h}$ at $37^{\circ} \mathrm{C}$ and $5 \%$ $\mathrm{CO}_{2}$, and was then replaced with either normal M199, with LACV diluted to $\sim 10^{3}$ PFUs/mL, or with M199 with gum tragacanth as above.

\section{LARVICIDAL ASSAYS}

Mosquitoes used were from a colony established from host-seeking A. albopictus collected in the Radford, Virginia, area, which has been maintained for 4 years with new field-collected adults added seasonally. This species was used because of its ease of laboratory colonization and because it is an efficient vector for a number of arboviruses, including LACV (Grimstad et al., 1989). All mosquito stages were maintained in an insectary held at $27^{\circ} \mathrm{C}$ and $80 \%$ relative humidity. Eggs were stimulated to hatch in $1.5 \mathrm{~L}$ deionized water containing $\sim 5 \mathrm{~mL}$ of a slurry of bovine liver powder $(9.375 \mathrm{~g} / \mathrm{L})$ and brewer's yeast $(3.125 \mathrm{~g} / \mathrm{L})$, which resulted in hatch within 1-2 days. Whole coffee extracts were serially twofold diluted from 1:2 to 1:8 in deionized water. Twenty-five A. albopictus larvae, mostly L1 but a few L2, were transferred 12 days post-hatching to $250-\mathrm{mL}$ glass beakers containing $100 \mathrm{~mL}$ of each coffee dilution $\left(50,25,12.5\right.$, or $\left.\mathrm{H}_{2} \mathrm{O}\right)$. Larvae were fed the bovine liver powder slurry as needed, generally $\sim 0.5 \mathrm{~mL}$ every other day. Surviving larvae were counted daily until all had died or pupated; a mosquito was considered living if it responded to gentle prodding with a transfer pipet. Pupae were removed from the beaker and were counted as surviving on all subsequent days, though we did not monitor survival of pupae and adults after their removal. Larvae reared in deionized water served as a control. Three independent replicates of each experiment were performed.

\section{ADULT MOSQUITO INFECTIONS}

To determine whether mosquitoes exposed to coffee during the larval stages have an altered susceptibility to LACV infection, we reared $\sim 100 \mathrm{~A}$. albopictus larvae in $1 \mathrm{~L}$ of either deionized water or $15 \%$ regular coffee, which induces only a moderate mortality in larvae. Mosquitoes were housed in $30 \mathrm{~cm} \times 30 \mathrm{~cm} \times 30 \mathrm{~cm}$ cages, allowed to freely mate, and given cotton balls soaked with $10 \%$ sucrose, which was removed $24 \mathrm{~h}$ prior to blood feeding. Approximately 7 days post-eclosion, adults were given a blood meal containing $5.82 \log _{10} \mathrm{PFU} / \mathrm{mL}$ LACV and held for 5 days at $27^{\circ} \mathrm{C}$ and $80 \%$ relative humidity. We selected the 5-day time point because the virus is not expected to have fully disseminated from the midgut (Paulson and Grimstad, 1989; Chandler et al., 1998), and therefore would pose only a minimal risk of accidental transmission. Mosquitoes that did not blood feed or only took a partial meal were destroyed. Individual mosquitoes were then homogenized in $50 \mu \mathrm{L}$ M199 using a plastic pestle, an additional $100 \mu \mathrm{L}$ M199 was added, and the homogenate was centrifuged $1 \mathrm{~min}$ at $14,000 \times g$ to pellet debris. The supernatant was serially 10-fold diluted in M199, and plaque assays were performed with $200 \mu \mathrm{L}$ of each dilution on Vero monolayers in 12-well plates (Costar) as described above, except antibiotic levels were increased to $125 \mu \mathrm{g} / 100 \mathrm{~mL}$ amphotericin $\mathrm{B}$ and $0.75 \mu \mathrm{g} / 100 \mathrm{~mL}$ gentamycin. Whole-body LACV titers were calculated for each mosquito.

\section{OVIPOSITION}

A small cohort of adult mosquitoes reared in 15\% coffee or in deionized water were allowed to take a non-infectious blood meal from a human arm. Those that fed to repletion were individually housed in $21 \mathrm{~mm} \times 70 \mathrm{~mm}$ shell vials containing $\sim 1 \mathrm{~cm}$ water and a strip of moist seed germination paper on which to oviposit. After 4 days, the number of eggs was counted under a dissecting microscope. Individuals that did not oviposit were discarded from the analysis.

\section{STATISTICAL ANALYSES}

All analyses were performed using JMP software (SAS Institute, Cary, NC, USA). Significance of antiviral assays was determined by ANOVA with Dunnett's test performed post hoc, comparing coffeeexposed virus levels to the virus control which was set to $100 \%$. Differences in larval survival following exposure to coffee dilutions were identified by Wilcoxon comparison of Kaplan-Meier survival curves. Individuals that pupated were censored at the date when all larvae had either pupated or died. When the Wilcoxon test among groups was significant, pairwise comparisons were made to the control group. Mosquito infections were compared by unpaired $t$-test of log-transformed whole-body titers. Egg numbers were compared by unpaired $t$-test.

\section{RESULTS ANTIVIRAL ASSAYS}

When regular Maxwell House Dark Roast coffee was incubated with LACV, direct antiviral activity was evident, and this activity was lost upon dilution (Figure 1). The coffee extract significantly reduced virus viability at final concentrations between 1.25 and $20 \%$, but not at $0.625 \%$. Similarly, Great Value 

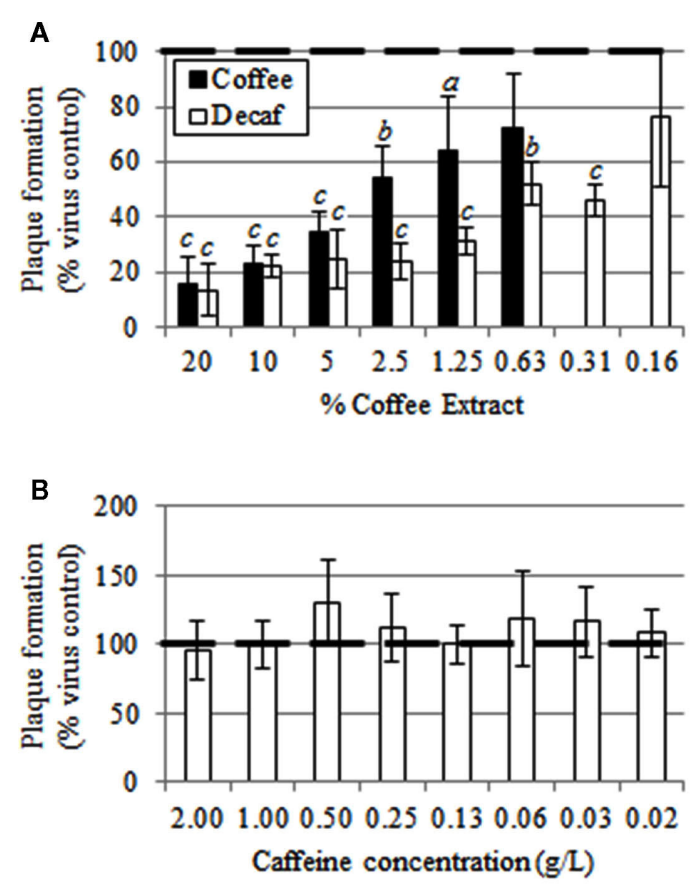

FIGURE 1 | (A) Direct anti-La Crosse virus effect of regular (black bars) and decaffeinated (white bars) coffee. (B) Lack of a direct anti-La Crosse virus effect upon exposure to caffeine. Error bars represent the SD of three independent replicates. $a: p<0.05 ; b: p<0.01$; $c$ : $p<0.0001$. The heavy, dashed, black line indicates the virus control, which was set to $100 \%$.

brand decaffeinated coffee exhibited strong antiviral effect; virus viability was significantly reduced at concentrations of $0.625-20 \%$ ( $p \leq 0.0002$ ), while the $0.15625 \%$ level was marginally significant $(p=0.0840$; Figure 1A). Thus, it is likely not the caffeine that has the antiviral activity, which was verified by the lack of virucidal activity seen with pure caffeine (Figure 1B). No toxic effects of the coffee on the Vero cells were noted.

Coffee extracts did not interfere with LACV replication when added to Vero cells $24 \mathrm{~h}$ before $(p=0.8105, t=0.2561)$ or $24 \mathrm{~h}$ after $(p=0.0777, t=2.3590)$ infection with LACV. When Vero cells were exposed to coffee extracts $1 \mathrm{~h}$ before infection, the production of LACV plaques was reduced by $52.5 \% \quad(p=0.0002$, $t=13.2500)$. Similarly, a $23.8 \%$ reduction in plaques was evident when coffee was added $1 \mathrm{~h}$ after LACV infection $(p=0.0200$, $t=3.7482$; data not shown).

\section{LARVICIDAL ACTIVITY}

Mosquitoes reared in 25 or 50\% regular coffee did not survive to pupation, and both concentrations exhibited similar survival curves; those reared in $12.5 \%$ coffee showed reduced mortality (Figure 2A). All pairwise comparisons were significantly different at $p \leq 0.0002$, with the exception of 50 vs. $25 \%(p=0.1320)$. Decaffeinated coffee was largely lethal at all concentrations tested, though the $50 \%$ concentration surprisingly allowed a few mosquitoes to pupate while the lower concentrations killed all larvae (Figure 2B). All pairwise comparisons were significantly different at $p<0.0001$. In contrast to the antiviral results, caffeine showed

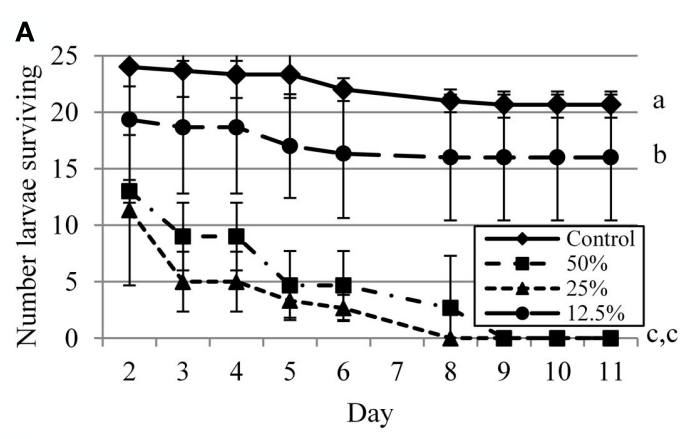

B

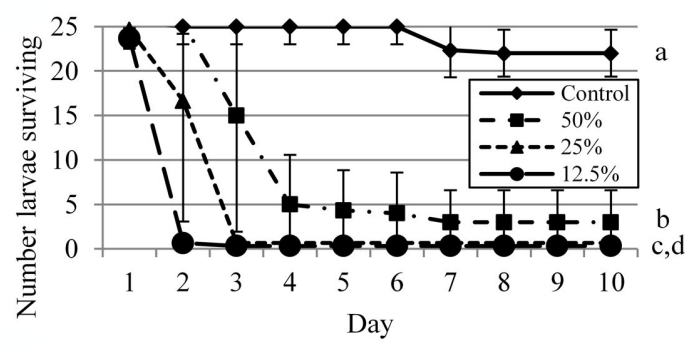

C

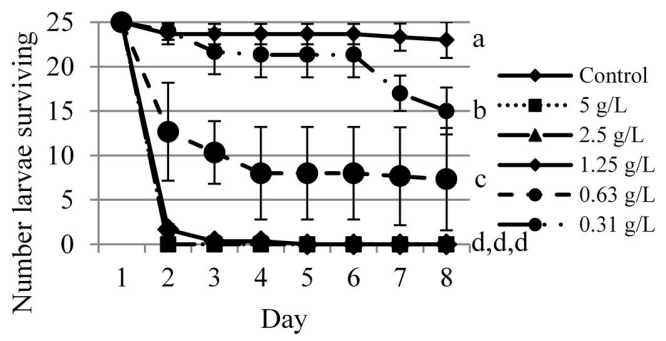

FIGURE 2 | Survival curves for Aedes albopictus larvae exposed to varying concentrations of regular coffee (A), decaffeinated coffee (B), or caffeine (C). Curves followed by different letters are significantly different $(p<0.05$ ) by Wilcoxon analysis of Kaplan-Meier survival curves.

relatively strong larvicidal action, especially at concentrations $\geq 1.25 \mathrm{~g} / \mathrm{L}$ (Figure 2C). All pairwise comparisons were significantly different at $p<0.001$, except 1.25 vs. 2.5 and $5.0 \mathrm{~g} / \mathrm{L}$ $(p=0.1739)$. We noted, but did not quantify here, that mosquitoes reared in coffee tended to pupate more quickly than water-reared controls.

\section{MODULATION OF VIRAL TITERS IN ADULT MOSOUITOES}

We reared mosquitoes in $15 \%$ coffee extract to yield sufficient surviving individuals to infect with LACV, yet be exposed to a relatively high concentration of coffee. These mosquitoes were then infected with a LACV-containing blood meal, and wholebody titers were determined after 5 days' infection. Exposure to coffee during the larval stages resulted in a significant reduction in the whole-body titer of mosquitoes infected with LACV as adults $(p=0.0090 ; t=2.780)$. Mosquitoes reared in distilled water $(N=17)$ had an average titer of $4.65 \log _{10} \mathrm{PFU} / \mathrm{mosquito}$, while those reared in coffee $(N=42)$ had a titer of $3.07 \log _{10}$ PFU/mosquito (Figure 3). Differences in body size were not obvious, though we did not measure wing lengths to verify this. 


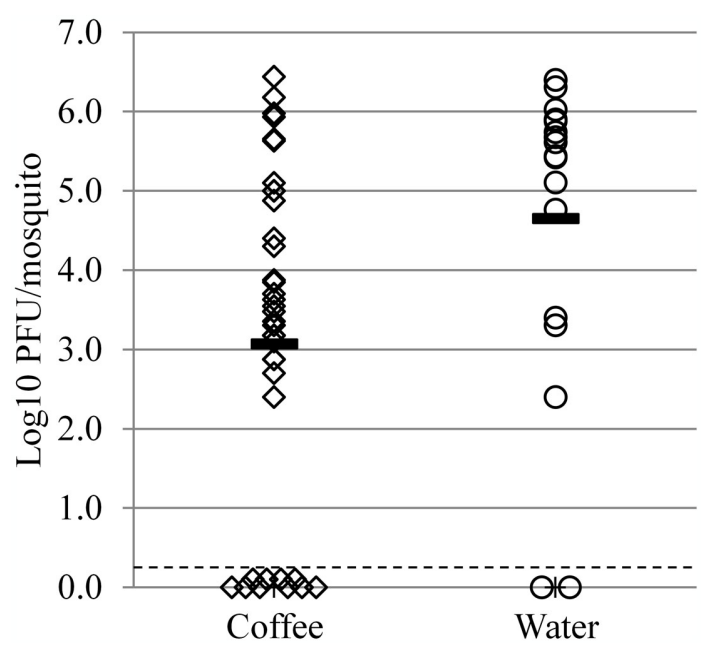

FIGURE 3 | Full-body La Crosse virus titers of Aedes albopictus females reared in $15 \%$ coffee extract (diamonds) or distilled water (circles). Solid horizontal lines indicate the mean for each group. Dashed line represents the limit of detection. Means are significantly different by $t$-test $(p=0.0090)$.

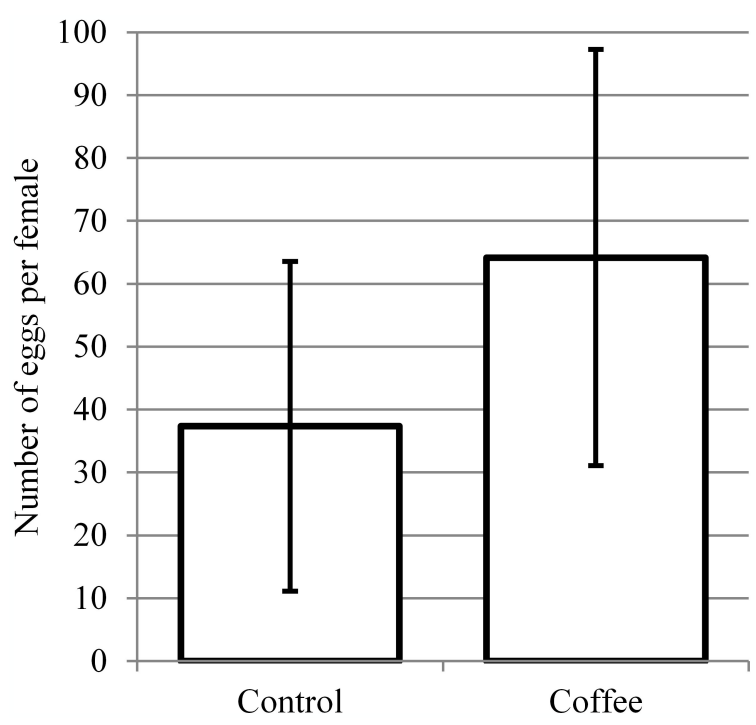

FIGURE 4 | Oviposition by females reared in water $(N=12)$ or $15 \%$ coffee $(\boldsymbol{N}=\mathbf{1 3})$. Means are significantly different by $t$-test $(p=0.0356)$.

\section{OVIPOSITION}

Females reared in $15 \%$ coffee $(N=12)$ laid an average of 64.2 eggs (range 1-92), whereas those reared in water $(N=13)$ deposited a mean of 37.3 eggs (range 11-113; Figure 4). This difference was significant $(p=0.0356 ; t=2.2327)$.

\section{DISCUSSION}

We present here a demonstration that it is possible to modify a mosquito's vector competence following exposure to an antiviral compound during the larval stages of its development. High concentrations of coffee were required to demonstrate antiviral and larvicidal activity individually and, thus, coffee extracts are likely not suitable as part of a wide-scale larvicidal and antiviral campaign unless the active components can be isolated. One such compound, pyridinium formate, has been shown to possess antiviral activity (Tsujimoto et al., 2010). Others may eventually be isolated, though, as $>700$ chemicals have been identified in extracts of roasted coffee beans (Spiller, 1984). Given the large number of compounds found in coffee, it is also improbable that the same compound is acting against both virus and larvae.

The mechanism by which coffee extracts exert their mode of action is unclear. We did not observe cytopathic effect in the Vero cells following the 1-h incubations with coffee extract. Hence, the coffee must be acting directly on the virus prior to our infection of the cell layers. In this case, the coffee could be inactivating the virus or interfering with an early step in the virus life cycle. The reduction in plaque numbers seen when coffee was added $1 \mathrm{~h}$, but not $24 \mathrm{~h}$, before or after virus infection suggests that an early step in the life cycle is targeted. A similar early inhibition was seen in cells infected with herpes simplex 1 virus (a virus with a DNA genome) following addition of coffee (Utsunomiya et al., 2008).

In these studies, decaffeinated coffee extract demonstrated a somewhat higher larvicidal activity and antiviral activity. This was unexpected, as previous experiments had shown similar herpesvirus inactivation rates using coffee extracts from broad geographic areas and between caffeinated and decaffeinated products, though these rates were quite variable among different brands (Utsunomiya et al., 2008). It is unlikely that the ethyl acetate method used to decaffeinate the coffee beans contributes to the increased larvicidal and antiviral activities, because the ethyl acetate is thoroughly removed during the process (Ramalakshmi and Raghavan, 1999). The variation seen between these two brands and formulations of coffee is most probably due to the different origins and degree of roasting of the coffee beans that may contain different concentrations of whatever compound(s) has the antiviral and/or larvicidal activity. Such variation in coffee compounds that have antibacterial activity has been shown among different coffee brands (Almeida et al., 2006). However, we did not attempt to identify or quantify the specific active compounds in this study.

Mosquito control using synthetic chemicals has been very successful, but resistance has developed to all insecticides used in their control (Hemingway et al., 2004). Bacterial toxins, such as those produced by Bacillus thuringiensis israelensis (Bti), have also proven useful. Again, resistance has begun to become evident (e.g., Paris et al., 2011), though not to the extent seen with insecticides. Plant extracts, such as from coffee, could potentially be used as control agents (Shaalan et al., 2005; Fallatah and Khater, 2010), though the likelihood of these being developed seems somewhat remote given the cost and regulatory burden associated with bringing new products to market (Isman, 2006).

We have documented several altered traits that can influence the vectorial capacity, or likelihood of transmission, of LACV. Vectorial capacity incorporates measures of vector density, feeding preference, survival, and extrinsic incubation period (EIP), which is the time it takes a mosquito to become infectious following a virus-containing blood meal (Black and Moore, 2005). Larval exposure to coffee extracts did not completely prevent LACV 
infection of $A$. albopictus, but did significantly reduce the virus titer in the mosquitoes. This could result in a lengthening of the EIP, the time it takes a mosquito to become infectious. Because the EIP interacts with the probability of daily survival exponentially, a small change in the EIP can have a dramatic effect on the likelihood of transmission (Anderson and Rico-Hesse, 2006). We noted that females reared in coffee tended to lay more eggs than control females, which could theoretically lead to a higher risk of virus transmission by increasing the vector density. Other measures implicit in determining vectorial capacity, such as coffee's influence on adult survival or fertility, remain to be assessed. Additionally, variation in the size of the mosquito also influences the likelihood of transmission, with smaller mosquitoes transmitting at higher rates (Paulson and Hawley, 1991; Anderson et al., 2005). This does not mean that virus titers vary, as A. albopictus body size does not influence the whole-body titer of dengue virus (Alto et al., 2008).

The exact nature of this resistance to infection remains to be determined, and it is unclear whether the antiviral activity seen in cell culture is actually occurring in live mosquitoes. Two mechanisms described in the literature seem possible and warrant further study following identification of the active compound(s). First, the active antiviral component of coffee may be integrated into the adult mosquito from the aquatic medium in which the larvae and pupae develop. Such carry-over from larvae to adult has been demonstrated by feeding larvae suspensions containing radioactive rubidium (Wilkins et al., 2007) or zinc (Lang, 1963); these isotopes are then detectible in emergent adults. Further, exposure to sublethal concentrations of growth regulators during the larval stages continues to have detrimental effects on the adults that survive exposure (Vasuki, 1992; Suman et al., 2010). Thus, the antiviral compound(s) may be carried over from larvae to adult and reduce LACV infectivity in the mosquito. The fact that Vero cells exposed to coffee $24 \mathrm{~h}$ before LACV infection do not yield a reduction in PFUs provide evidence against this possibility, however. Second,

\section{REFERENCES}

Almeida, A. A., Farah, A., Silva, D. A., Nunan, E. A., and Gloria, M. B. (2006). Antibacterial activity of coffee extracts and selected coffee chemical compounds against enterobacteria. J. Agric. Food Chem. 54, 8738-8743.

Alto, B. W., Reiskind, M. H., and Lounibos, L. P. (2008). Size alters susceptibility of vectors to dengue virus infection and dissemination. Am. J. Trop. Med. Hyg. 79, 688-695.

Anderson, J. R., and Rico-Hesse, R. (2006). Aedes aegypti vectorial capacity is determined by the infecting genotype of dengue virus. Am. J. Trop. Med. Hyg. 75, 886-892.

Anderson, J. R., Schneider, J. R., Grimstad, P. R., and Severson, D. W. (2005). Quantitative genetics of vector competence for La Crosse virus and body size in Ochlerotatus hendersoni and Ochlerotatus triseriatus interspecific hybrids. Genetics 169, 1529-1539.

Black, W. C. IV, and Moore, C. G. (2005). "Population biology as a tool to study vector-borne diseases," in The Biology of Disease Vectors, eds W. C. Marquardt, W. C. Black IV, S. Higgs, J. E. Freier, A. A. James, H. H. Hagedorn, B. Kondratieff, J. Hemingway, and C. G. Moore (Burlington, MA: Elsevier Academic Press), 187-206.

Borucki, M. K., Kempf, B. J., Blitvich, B. J., Blair, C. D., and Beaty, B. J. (2002). La Crosse virus: replication in vertebrate and invertebrate hosts. Microbes Infect. 4, 341-350.

Chandler, L. J., Blair, C. D., and Beaty, B. J. (1998). La Crosse virus infection of Aedes triseriatus (Diptera: Culicidae) ovaries before dissemination of virus from the midgut. J. Med. Entomol. 35, 567-572.

Cui, F., Raymond, M., and Qiao, C. L. (2006). Insecticide resistance in

exposure to coffee compounds may increase expression of stressrelated genes, which may include those involved in the insect's innate immune system. Accordingly, A. aegypti larvae exposed to used coffee grounds and caffeine showed altered patterns of esterase expression compared to water-reared controls (Laranja et al., 2003). Daphnia magna Straus exposed to the herbicide propanil up-regulate a number of stress proteins (Pereira et al., 2010), as do Drosophila melanogaster (Meigen) when exposed to the pesticide endosulfan (Sharma et al., 2011). Such proteins may influence arbovirus replication in mosquitoes. For example, proteins involved in the Toll (Xi et al., 2008; Ramirez and Dimopoulos, 2010) and JAK-STAT (Souza-Neto et al., 2009) pathways influence the development of dengue virus infection in A. aegypti mosquitoes. However, the exact mode of action remains to be determined and refined.

In conclusion, we have demonstrated that some brands of coffee extracts have both mosquito larvicidal and anti-LACV activity, and we have further shown that replication of LACV can be modulated in adult mosquitoes by exposing the larvae to coffee extracts. Our results are at least a proof of concept, and we did not identify the actual active agent(s) responsible for our observed effects. However, this represents a potential new way to interfere with arbovirus transmission at two steps in the virus's life cycle, namely by decreasing the number of vectors and by reducing or blocking the replication of the virus in vector mosquitoes exposed to the virus. The large number of antiviral plant-derived compounds deserves further study to develop this mechanism into an effective arbovirus-control strategy.

\section{ACKNOWLEDGMENTS}

Funding was provided by a Radford University Seed Grant to Justin R. Anderson, by the RU Department of Biology, and by the Rogers Undergraduate Research Award to Nicole E. Eastep (2009) and Rachel E. Albert (2010). Amanda Robinson and Kimberly Filcek assisted with plaque assays.

vector mosquitoes in China. Pest Manag. Sci. 62, 1013-1022.

Fallatah, S. A., and Khater, E. I. (2010). Potential of medicinal plants in mosquito control. J. Egypt. Soc. Parasitol. 40, 1-26.

Grimstad, P. R., Kobayashi, J. F., Zhang, M. B., and Craig, G. B. Jr. (1989). Recently introduced Aedes albopictus in the United States: potential vector of La Crosse virus (Bunyaviridae: California serogroup). J. Am. Mosq. Control Assoc. 5, 422-427.

Haddow, A. D., and Odoi, A. (2009). The incidence risk, clustering, and clinical presentation of La Crosse virus infections in the eastern United States, 2003-2007. PLoS ONE 4, e6145. doi:10.1371/journal.pone.0006145

Hemingway, J., Hawkes, N. J., McCarroll, L., and Ranson, H. (2004). The molecular basis of insecticide resistance in mosquitoes. Insect Biochem. Mol. Biol. 34, 653-665.
Isman, M. B. (2006). Botanical insecticides, deterrents, and repellents in modern agriculture and an increasingly regulated world. Annu. Rev. Entomol. 51, 45-66.

Jassim, S. A., and Naji, M. A. (2003). Novel antiviral agents: a medicinal plant perspective. J. Appl. Microbiol. 95, 412-427.

Lambrechts, L., Knox, T. B., Wong, J., Liebman, K. A., Albright, R. G., and Stoddard, S. T. (2009). Shifting priorities in vector biology to improve control of vector-borne disease. Trop. Med. Int. Health 14, 1505-1514.

Lang, C. A. (1963). The accumulation of zinc by the mosquito. J. Gen. Physiol. 46, 617-627.

Laranja, A. T., Manzato, A. J., and Bicudo, H. E. (2003). Effects of caffeine and used coffee grounds on biological features of Aedes aegypti (Diptera: Culicidae) and their possible use in alternative 
control. Genet. Mol. Biol. 26, 419-429.

Laranja, A. T., Manzato, A. J., and Bicudo, H. E. (2006). Caffeine effect on mortality and oviposition in successive generations of Aedes aegypti. Rev. Saude. Publica 40, 1112-1117.

Luckhart, S., Lindsay, S. W., James, A. A., and Scott, T. W. (2010). Reframing critical needs in vector biology and management of vector-borne disease. PLoS Negl. Trop. Dis. 4, e566. doi:10.1371/journal.pntd.0000566

Mukhtar, M., Arshad, M., Ahmad, M., Pomerantz, R. J., Wigdahl, B., and Parveen, Z. (2008). Antiviral potentials of medicinal plants. Virus Res. 131, 111-120.

Murayama, M., Tsujimoto, K., Uozaki, M., Katsuyama, Y., Yamasaki, H., Utsunomiya, H., and Koyama, A. H. (2008). Effect of caffeine on the multiplication of DNA and RNA viruses. Mol. Med. Report 1, 251-255.

Paris, M., Tetreau, G., Laurent, F., Lelu, M., Despres, L., and David, J. P. (2011). Persistence of Bacillus thuringiensis israelensis (bti) in the environment induces resistance to multiple bti toxins in mosquitoes. Pest Manag. Sci. 67, 122-128.

Paulson, S. L., and Grimstad, P. R. (1989). Replication and dissemination of La Crosse virus in the competent vector Aedes triseriatus and the incompetent vector Aedes hendersoni and evidence for transovarial transmission by Aedes hendersoni (Diptera: Culicidae). J. Med. Entomol. 26, 602-609.
Paulson, S. L., and Hawley, W. A. (1991). Effect of body size on the vector competence of field and laboratory populations of Aedes triseriatus for La Crosse virus. J. Am. Mosq. Control Assoc. 7, 170-175.

Pereira, J. L., Hill, C. J., Sibly, R. M., Bolshakov, V. N., Goncalves, F., Heckmann, L. H., and Callaghan, A. (2010). Gene transcription in Daphnia magna: effects of acute exposure to a carbamate insecticide and an acetanilide herbicide. Aquat. Toxicol. 97, 268-276.

Ramalakshmi, K., and Raghavan, B. (1999). Caffeine in coffee: its removal. why and how? Crit. Rev. Food Sci. Nutr. 39, 441-456.

Ramirez, J. L., and Dimopoulos, G. (2010). The toll immune signaling pathway control conserved antidengue defenses across diverse Ae. aegypti strains and against multiple dengue virus serotypes. Dev. Comp. Immunol. 34, 625-629.

Sardelis, M. R., Turell, M. J., and Andre, R. G. (2002). Laboratory transmission of La Crosse virus by Ochlerotatus japonicus (Diptera: Culicidae). J. Med. Entomol. 39, 635-639.

Shaalan, E. A., Canyon, D., Younes, M. W., Abdel-Wahab, H., and Mansour, A. H. (2005). A review of botanical phytochemicals with mosquitocidal potential. Environ. Int. 31, 1149-1166.

Sharma, A., Mishra, M., Ram, K. R., Kumar, R., Abdin, M. Z., and Chowdhuri, D. K. (2011). Transcriptome analysis provides insights for understanding the adverse effects of endosulfan in Drosophila melanogaster. Chemosphere 82, 370-376.

Souza-Neto, J. A., Sim, S., and Dimopoulos, G. (2009). An evolutionary conserved function of the JAK-STAT pathway in anti-dengue defense. Proc. Natl. Acad. Sci. U.S.A. 106, 17841-17846.

Spiller, M.A. (1984). The chemical components of coffee. Prog. Clin. Biol. Res. 158, 91-147.

Suman, D. S., Parashar, B. D., and Prakash, S. (2010). Effect of sublethal dose of diflubenzuron and azadirachtin on various life table attributes of Culex quinquefasciatus (Diptera: Culicidae). J. Med. Entomol. 47, 996-1002.

Tsujimoto, K., Sakuma, C., Uozaki, M., Yamasaki, H., Utsunomiya, H., Oka, K., and Koyama, A. H. (2010) Antiviral effect of pyridinium formate, a novel component of coffee extracts. Int. J. Mol. Med. 25, 459-463.

Utsunomiya, H., Ichinose, M., Uozaki, M., Tsujimoto, K., Yamasaki, H., and Koyama, A. H. (2008). Antiviral activities of coffee extracts in vitro. Food Chem. Toxicol. 46, 1919-1924.

Vasuki, V. (1992). Adult longevity of certain mosquito species after larval and pupal exposure to sublethal concentration of an insect growth regulator, hexaflumuron. Southeas Asian J. Trop. Med. Public Health 23, 121-124.
Wilkins, E. E., Smith, S. C., Roberts, J. M., and Benedict, M. (2007). Rubidium marking of anopheles mosquitoes detectable by field-capable Xray spectrometry. Med. Vet. Entomol. 21, 196-203.

Xi, Z., Ramirez, J. L., and Dimopoulos, G. (2008). The Aedes aegypti toll pathway controls dengue virus infection. PLoS Pathog. 4, e1000098. doi:10.1371/journal.ppat. 1000098

Conflict of Interest Statement: The authors declare that the research was conducted in the absence of any commercial or financial relationships that could be construed as a potential conflict of interest.

Received: 10 November 2011; accepted: 07 March 2012; published online: 28 March 2012.

Citation: Eastep NE, Albert RE and Anderson JR (2012) Modulation of La Crosse virus infection in Aedes albopictus mosquitoes following larval exposure to coffee extracts. Front. Physio. 3:66. doi: 10.3389/fphys.2012.00066

This article was submitted to Frontiers in Systems Biology, a specialty of Frontiers in Physiology.

Copyright (c) 2012 Eastep, Albert and Anderson. This is an open-access article distributed under the terms of the Creative Commons Attribution Non Commercial License, which permits noncommercial use, distribution, and reproduction in other forums, provided the original authors and source are credited. 